\title{
Feasibility of oxygen sensitive cardiac magnetic resonance of the right ventricle in pulmonary artery hypertension
}

\author{
Karthigesh Sree Raman ${ }^{1,2,3}$, Michael Stokes ${ }^{4}$, Angela Walls ${ }^{5}$, Rebecca Perry ${ }^{1,2,3}$, Peter M. Steele ${ }^{4}$, \\ Christine Burdeniuk ${ }^{2}$, Carmine G. De Pasquale ${ }^{1,2}$, David S. Celermajer ${ }^{6,7}$, Joseph B. Selvanayagam ${ }^{1,2,3}$ \\ ${ }^{1}$ College of Medicine and Public Health, Flinders University, Adelaide, Australia; ${ }^{2}$ Department of Cardiovascular Medicine, Flinders Medical \\ Centre, Adelaide, Australia; ${ }^{3}$ Cardiac Imaging Research, South Australian Health \& Medical Research Institute, Adelaide, Australia; ${ }^{4}$ Department \\ of Cardiology, Royal Adelaide Hospital, Adelaide, Australia; ${ }^{5}$ Clinical Research and Imaging Centre, South Australian Health \& Medical Research \\ Institute, Adelaide, Australia; ${ }^{6}$ Sydney Medical School, University of Sydney and Royal Prince Alfred Hospital, Sydney, Australia; ${ }^{7}$ Department of \\ Cardiology, Royal Prince Alfred Hospital, Sydney, Australia \\ Contributions: (I) Conception and design: DS Celermajer, JB Selvanayagam; (II) Administrative support: K Sree Raman, R Perry; (III) Provision of study \\ materials or patients: M Stokes, PM Steele, C Burdeniuk, CG De Pasquale; (IV) Collection and assembly of data: K Sree Raman, A Walls; (V) Data \\ analysis and interpretation: K Sree Raman, JB Selvanayagam; (VI) Manuscript writing: All authors; (VII) Final approval of manuscript: All authors. \\ Correspondence to: Professor Joseph B. Selvanayagam, DPhil. Department of Cardiovascular Medicine, Flinders Medical Centre, Adelaide, South \\ Australia 5042, Australia. Email: Joseph.Selvanayagam@flinders.edu.au.
}

Background: Progressive right ventricular (RV) dysfunction in pulmonary arterial hypertension (PAH) which is contributed by RV ischemia leads to adverse clinical outcomes. Oxygen-sensitive (OS) cardiovascular magnetic resonance (CMR) has been used to determine the in vivo myocardial oxygenation of the left ventricle $(\mathrm{LV})$. The aims of the present study were therefore to determine the feasibility of RV targeted rest/ stress OS-CMR imaging in PAH patients and healthy volunteers.

Methods: We prospectively recruited 20 patients with right heart catheter proven PAH and 9 healthy age matched controls (NC). The CMR examination involved standard functional imaging and OS-CMR imaging. An OS-CMR signal intensity (SI) index (stress/rest SI) was acquired at RV anterior, RV free-wall and RV inferior segments. In the LV, the OS-CMR SI index was acquired globally.

Results: Reliable OS SI changes were only obtained from the RV inferior segment. As RV dysfunction in $\mathrm{PAH}$ is a global process, hence this segment was used in both patients and $\mathrm{NC}$ for further comparison. RV OS-CMR SI change between rest and stress in the NC was $17 \% \pm 5 \%$ (mean \pm SD). Nine of $20(45 \%)$ of the PAH patients had a mean OS SI change of less than $9 \%$ (or $\geq 2$ SD different from the mean values in NC). Overall, RV OS SI index between the PAH patients and NC was $11 \% \pm 9 \%$ vs. $17 \% \pm 5 \%(\mathrm{P}=0.045)$ in the RV inferior segment. In the LV, the global OS-CMR SI index between the PAH patients and NC was $11 \% \pm 7 \%$ vs. $21 \% \pm 9 \%(\mathrm{P}=0.019)$. There was a strong correlation between RV Inf OS-CMR SI and LV OS-CMR SI $(\mathrm{r}=0.86, \mathrm{P}<0.001)$.

Conclusions: In this small pilot study, pharmacological induced OS-CMR is a feasible and safe technique to identify and study myocardial oxygenation in the RV of PAH patients.

Keywords: Pulmonary artery hypertension; cardiac magnetic resonance (CMR); microcirculation; microvascular ischemia; oxygen-sensitive cardiac magnetic resonance

Submitted Jul 10, 2019. Accepted for publication Sep 17, 2019.

doi: $10.21037 /$ cdt.2019.09.19

View this article at: http://dx.doi.org/10.21037/cdt.2019.09.19 


\section{Introduction}

Pulmonary arterial hypertension $(\mathrm{PAH})$ is a progressive disorder that affects both the pulmonary vasculature and the heart (1). The natural progression of $\mathrm{PAH}$ involves gradual development of right ventricular (RV) dysfunction which is often silent until advanced $(2,3)$. Whilst the exact cause of RV remodelling and subsequent dysfunction remains unclear; an ischemic precipitant is thought to be a significant contributor (4). Oxygen-sensitive (OS) cardiovascular magnetic resonance (CMR) technique has been used to investigate myocardial oxygenation and ischemia in the left ventricle (LV) $(5,6)$. This technique has been based on the effects of oxygenation on the paramagnetic properties of haemoglobin (7). Deoxygenated haemoglobin acts as an endogenous contrast agent with $\mathrm{T}_{2} / \mathrm{T}_{2}{ }^{*}$-dependent signal effects. The signal intensity (SI) changes in OS-CMR images are inversely correlated with the concentration of deoxygenated haemoglobin. Hence with the relative increase of deoxygenated haemoglobin there will be a decrease in OS-CMR SI. Although OSCMR has been used to advance the understanding of myocardial ischemia and a number of LV myocardial (8-11) and coronary artery disease states $(6,12,13)$, the utility in the RV has not yet been determined. Additionally, the need for other markers of early recognition and diagnosis of PAH is paramount, particularly for individuals at higher risk for development of RV failure. It is possible that the novel imaging technique of RV targeted OS-CMR may provide an earlier diagnosis of RV abnormality in these patients. However, before the utility of these techniques can be implemented as a screening tool for RV ischemia and/ or dysfunction, the feasibility of this technique must first be established. The aim of the present work therefore was to examine the feasibility of RV targeted OS-CMR to detect ischemia in a population with known $\mathrm{PAH}$.

\section{Methods}

\section{Study population}

Patients undergoing treatment in the PAH clinics at two South Australian hospitals were invited to participate in this study. Patients were considered for inclusion if they had right heart catheter proven $\mathrm{PAH}$ and resting echocardiographic mean PA pressure between $25-50 \mathrm{mmHg}$ (as measured by the peak pulmonary regurgitant signal on echocardiography and added to estimated right atrial pressure) and/or systolic PA pressure between $40-90 \mathrm{mmHg}$ (as measured by the peak tricuspid regurgitant velocity gradient on echocardiography and added to estimated right atrial pressure). Exclusion criteria included severe RV dysfunction on echocardiography, diagnosis of significant left heart cardiomyopathy and/or coronary artery disease (defined as $>70 \%$ luminal stenosis in an epicardial coronary artery at angiography or prior myocardial infarction) as well as contraindications to CMR and/or adenosine (second or third degree heart block, obstructive pulmonary disease or dipyridamole use). Nine healthy volunteers also underwent the same imaging procedures to act as a control group. The healthy volunteers had no known cardiac disease or symptoms and were free of any cardiac risk factors, including hypertension, smoking or diabetes. This study was approved by the Southern Adelaide Clinical Human Research Ethics Committee, and all participants provided written and informed consent to participate in the study.

\section{CMR protocol}

Participants were scanned using a 3 Tesla clinical MR scanner (Siemens, 3T MAGNETOM Skyra, 18 channel torso phased array coil in conjunction with a spinal coil posteriorly) and refrained from caffeine 24 hours prior to the scan. The scanning protocol included Half-Fourier single-shot turbo spin echo (HASTE) and Fast imaging with steady precession (TrueFISP) localisers. Cine images were acquired in vertical and horizontal long-axis, and ten short-axis images covering the entire right and left ventricles, using a retrospective ECG gating steady-state free precession (SSFP) sequence [repetition time (TR) $3 \mathrm{~ms}$, echo time (TE) $1.5 \mathrm{~ms}$, matrix $173 \times 256$, field of view $370 \mathrm{~mm} \times 370 \mathrm{~mm}$, bandwidth 1,302 , spatial resolution $1.81 \times 1.45 \times 6$, slice thickness $6 \mathrm{~mm}$, flip angle $55^{\circ}$ ]. For OS imaging, a single mid-ventricular slice was acquired at mid-diastole using a single-shot T2-prepared ECG-gated SSFP sequence (TR $256 \mathrm{~ms}$, TE $1.21 \mathrm{~ms}$, T2 preparation time $40 \mathrm{~ms}$, matrix $168 \times 192$, field of view $340 \mathrm{~mm} \times$ $340 \mathrm{~mm}$, slice thickness $6 \mathrm{~mm}$, flip angle $44^{\circ}$ ). Volume shimming and frequency adjustments were performed as required before the oxygenation imaging to minimise offresonance artefacts. To optimise the OS-CMR for the RV, a frequency with the fewest banding artefacts and best homogeneity was selected and applied to the OS sequence. In addition, the volume, size and shape of the shim was set to tightly cover the heart in the mid short axis slice, 


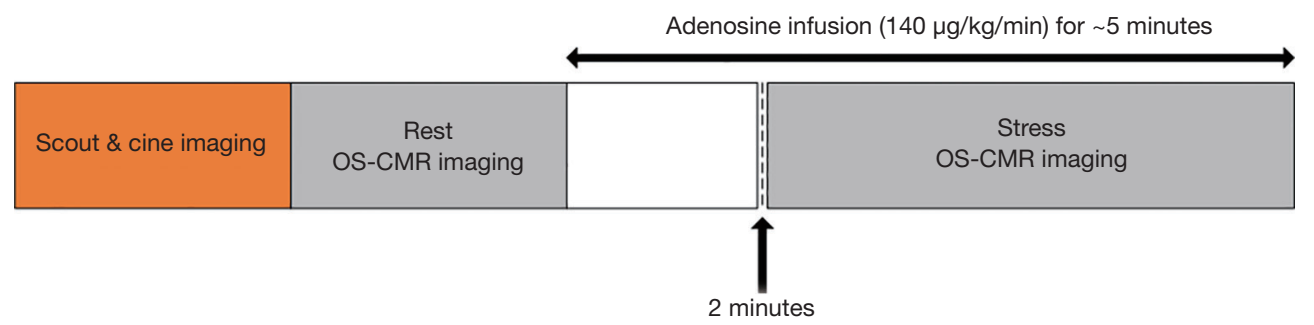

Figure 1 CMR Protocol. CMR, cardiovascular magnetic resonance.
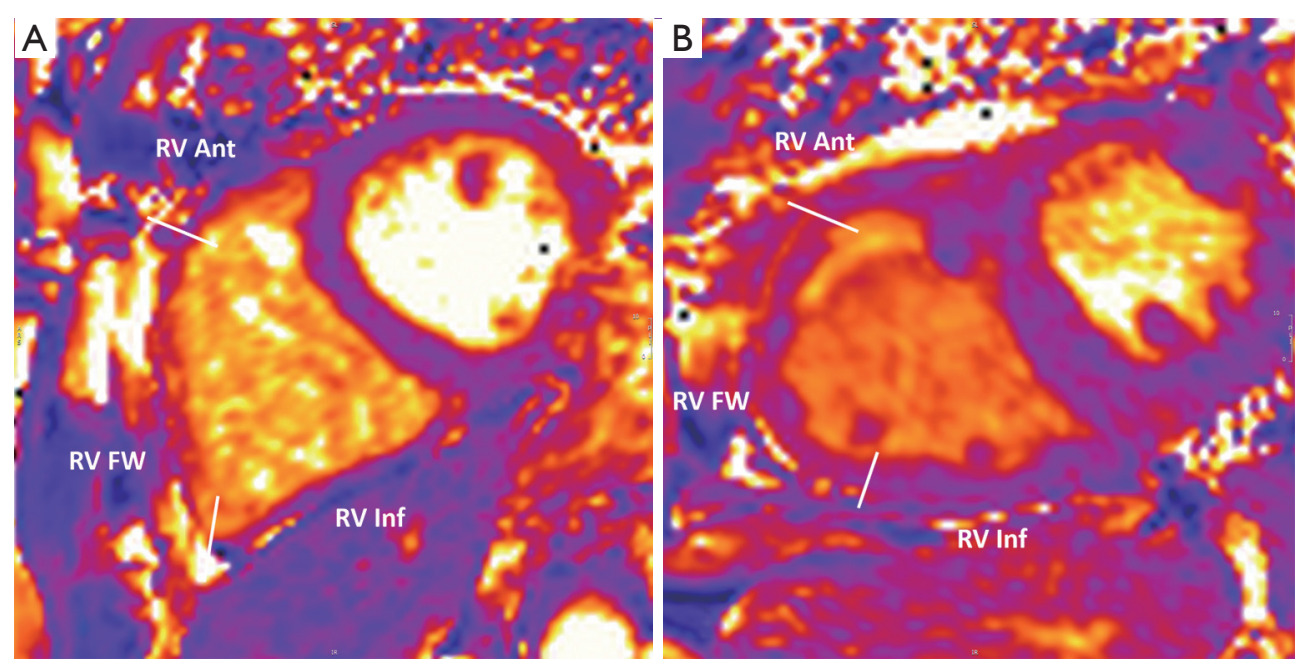

Figure 2 Colour Myo-map of OS-CMR signal intensity in a mid-ventricular short axis view demonstrating the delineation of RV Ant, RV FW and RF Inf segments in controls (A) and pulmonary hypertension patients (B). OS, oxygen-sensitive; CMR, cardiovascular magnetic resonance; RV Ant, right ventricle anterior; RV FW, right ventricle free-wall; RF Inf, right ventricle inferior.

with the thickness reduced to shim the slice area only. This enables further reduction of both banding frequency artefact and susceptibility artefacts. Each OS-CMR image was acquired during a single breath-hold over six heart beats. Four resting OS-CMR images were acquired at rest prior to commencement of adenosine infusion. Acquisition of the OS-CMR stress images commenced at 2 minutes of adenosine infusion. Each acquisition lasted 8-10 seconds on average (was heart rate dependent), we obtained four to six OS-CMR acquisitions over the stress period with an average of 30 seconds interval between acquisitions. Hence, typical duration of stress OS-CMR image acquisition was 3 minutes with the total duration of adenosine infusion was about 5 minutes (Figure 1). Stress heart rate and blood pressure were obtained every minute of adenosine infusion. Patients were monitored by ECG, sphygmomanometry and pulse oximetry throughout the study.

\section{CMR analysis}

The LV and RV function were analysed quantitatively from the short-axis SSFP images using $\mathrm{CVI}^{42}$ (Circle CVI, Calgary, Canada). Standard function parameters were calculated including $\mathrm{LV}$ ejection fraction (LVEF), RV ejection fraction (RVEF), cardiac index (CI), and body surface area indexed end-diastolic (EDVi), end-systolic (ESVi) volumes for LV/ $\mathrm{RV}$ and $\mathrm{LV}$ indexed myocardial mass.

For OS-CMR assessment of the RV, a region of interest (ROI) contour was traced manually using $\mathrm{CVI}^{42}$ (Circle CVI, Calgary, Canada). The RV was segmented to into $\mathrm{RV}$ anterior (RV Ant), RV free-wall (RV FW) and RV inferior (RV Inf) (Figure 2). OS-CMR assessment of the $\mathrm{LV}$ was performed whereby epicardium and endocardium borders were manually traced and was sub-divided into 6 equiangular segments based on a standard American Heart Association segmentation of the mid-ventricular slice (14). 
The mean resting myocardial signal intensity (SI) within each segment was obtained by averaging signal measurements from all the acquired rest OS-CMR images. Similarly, mean stress SI was calculated by averaging the signal measurements from all the stress OS-CMR images acquired during adenosine infusion. As it is a cardiac gated sequence, corrections have been made to the mean SI due to the variations of heart rate and its effects on T1 relaxation (15). These corrections have been applied to previous OS-CMR studies involving the $\mathrm{LV}$, and similar correction has been applied to the OS-CMR RV SI. The following equation was used for correction for measured SI for heart rate:

$$
\mathrm{S}=\mathrm{S}_{0} /\left[1-\beta \mathrm{e}^{(-\mathrm{TR} / \mathrm{T} 1)}\right]
$$

where $\mathrm{S}$ is the corrected SI and $\mathrm{S}_{0}$ is the measured SI. TR is the image dependent time between acquisitions of sections of $k$-space, governed by the heart rate. An empirical value of $\mathrm{T} 1=1,220 \mathrm{~ms}^{22}$ and $\beta=0.59$ from previously described work was appropriate for this sequence (12). The relative SI change was calculated as: $\Delta$ SI $(\%)=($ SI stress SI rest)/SI rest $\times 100$.

OS-CMR analysis of the $\mathrm{LV}$ was performed as previously described (12). As PAH is a global process the LV OS-CMR assessment was evaluated globally by averaging the $\Delta$ SI (\%) from all the $6 \mathrm{LV}$ segments.

All OS-CMR analysis was undertaken by experienced (SCMR Level 3) observers blinded to the clinical information of the participant. To evaluate interreproducibility, the OS-CMR images were independently assess by 2 experienced (SCMR Level 3) observers. To evaluate intra-observer reproducibility, one observer measured OS-CMR SI twice on 2 separate days with a washout period of at least 2 weeks.

\section{Statistical analysis}

Baseline and follow up data will be summarised using appropriate descriptive statistics and graphical representations. Discrete variables are summarised using the frequencies and percentages, whereas continuous variables will be presented by mean, standard deviation, median, Q1, Q3 as appropriate. Percentages will be calculated according to the number of patients for whom the data are available. These data will be presented for all patients and $\mathrm{P}$ value reported for the difference between the groups using the appropriate statistical tests for categorical or continuous variables. Statistical tests were two-tailed and $\mathrm{P}<0.05$ was considered statistically significant. Reproducibility of OSCMR SI measurements was assessed by using the coefficient variability method. Associations between the test parameters were assessed using the Pearson $r$ correlation coefficient.

\section{Results}

\section{Participants characteristics}

The baseline characteristics of both groups are detailed in Table 1. Both groups were well matched for age and gender, but the control group had a lower body mass index (BMI, 27.0 vs. $\left.30.9 \mathrm{~kg} / \mathrm{m}^{2}, \mathrm{P}=0.004\right)$. Amongst the $\mathrm{PAH}$ patients, $11(55 \%)$ had idiopathic pulmonary hypertension (iPAH), $6(30 \%)$ patients had systemic sclerosis (SSc)-associated PAH and $3(15 \%)$ patients had chronic thromboembolic PAH (CTEPH). All patients had right heart catheter proven diagnosis of $\mathrm{PAH}$ with a mean pulmonary artery pressure of 35 [30-41] $\mathrm{mmHg}$ and mean pulmonary capillary wedge pressure of 12 [10-14] mmHg. Mean 6-minute walk distance was 421 [368-474] meters. World Health Organisation functional class was between class II $(\mathrm{n}=16$, $80 \%)$ and III $(n=4,20 \%)$. On echocardiography seven patients (35\%) had RV dilatation but none had systolic RV dysfunction. The PAH patients had an echocardiographyestimated mean pulmonary artery pressure of 47 [38-57] mmHg. All PAH patients were treated with pulmonary vasodilators such as bosentan, macitentan, sildenafil, riociguat or a combination macitentan and sildenafil. The PAH patients were stable on treatment and continued their pulmonary vasodilators therapy during the OS-CMR research scan.

\section{CMR characteristics}

Table 2 shows a comparison of CMR variables between $\mathrm{PAH}$ patients and controls. The only statistically significant difference in CMR volumetric and functional indices between $\mathrm{PAH}$ and controls were the RV mass index $(20 \pm 12$ vs. $\left.14 \pm 9 \mathrm{~g} / \mathrm{m}^{2}, \mathrm{P}=0.014\right)$ and $\mathrm{RV}$ inferior wall thickness $(5 \pm 1$ vs. $4 \pm 1 \mathrm{~mm}, \mathrm{P}=0.019)$. The $\mathrm{RV}$ inferior wall thickness was measured on the SA cine slice location similar to the mid ventricular slice location chosen for the OS imaging.

\section{$R V$ myocardial oxygenation response (OS-CMR)}

\section{Safety and tolerability}

All participants tolerated and completed the adenosine induced stress component of the OS-CMR. Both the patients and controls had a good hemodynamic stress 
Table 1 Patient demographics and baseline clinical data

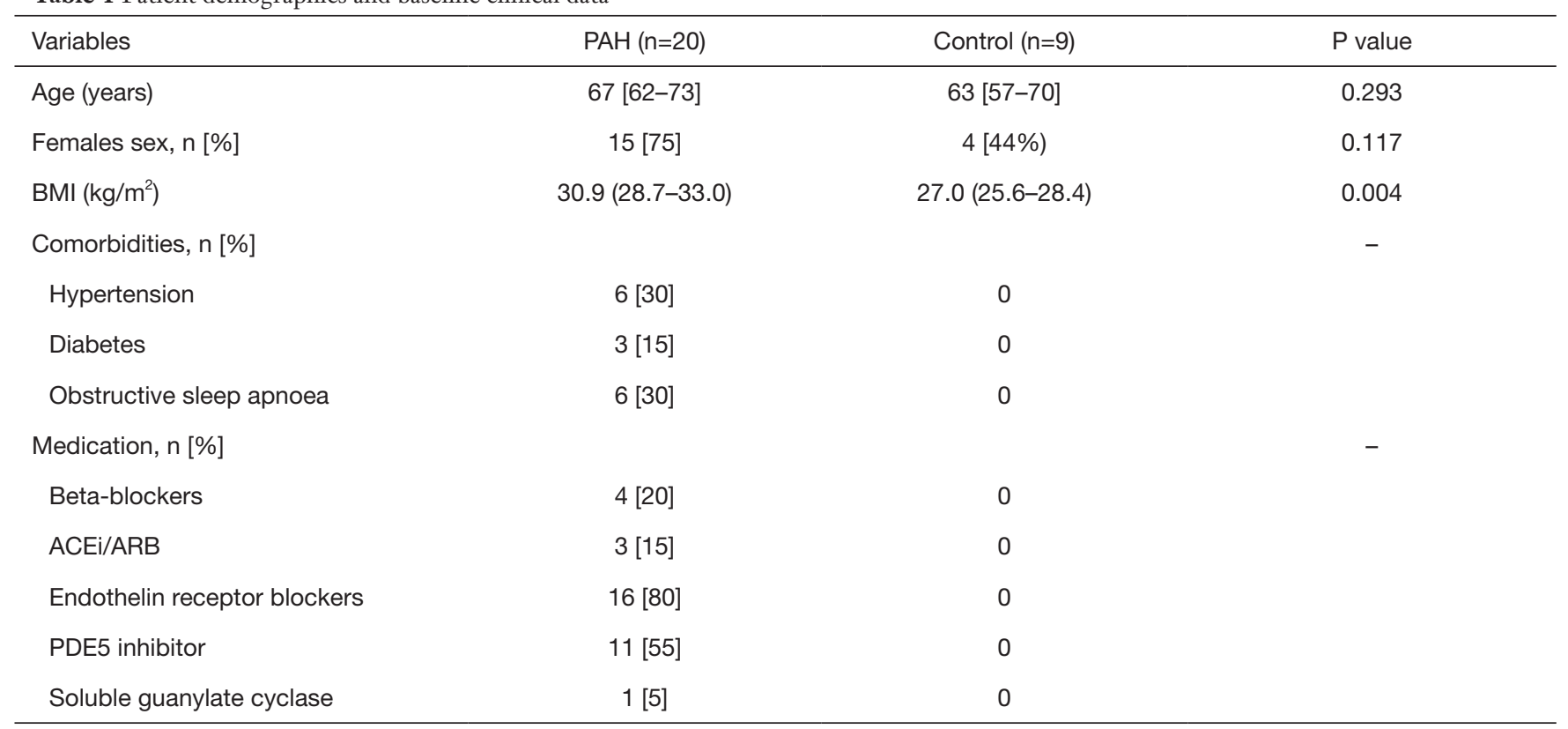

ACEi, angiotensin-converting enzyme inhibitor; ARB, angiotensin receptor blocker.

Table 2 CMR ventricular function

\begin{tabular}{|c|c|c|c|}
\hline Variables & $\mathrm{PAH}(\mathrm{n}=20)$, mean $\pm \mathrm{SD}$ & Control $(n=9)$, mean \pm SD & $P$ value \\
\hline CMR LV EDVi (mL/m²) & $62 \pm 24$ & $66 \pm 11$ & 0.505 \\
\hline CMR LV ESVi (mL/m²) & $21 \pm 11$ & $19 \pm 4$ & 0.496 \\
\hline CMR LV ED mass index $\left(\mathrm{g} / \mathrm{m}^{2}\right)$ & $48 \pm 18$ & $51 \pm 10$ & 0.593 \\
\hline CMR RVEF (\%) & $58 \pm 11$ & $64 \pm 9$ & 0.165 \\
\hline CMR RV EDVi (mL/m²) & $66 \pm 23$ & $65 \pm 14$ & 0.846 \\
\hline CMR RV ESVi $\left(\mathrm{mL} / \mathrm{m}^{2}\right)$ & $28 \pm 17$ & $24 \pm 9$ & 0.321 \\
\hline CMR RV SV index $\left(\mathrm{mL} / \mathrm{m}^{2}\right)$ & $38 \pm 12$ & $41 \pm 10$ & 0.412 \\
\hline
\end{tabular}

*, RV inferior wall was measured at the short axis cine slice location similar to the OS-CMR mid ventricular slice location. OS, oxygensensitive; CMR, cardiovascular magnetic resonance; RV, right ventricle; LV, left ventricle; LVEF, LV ejection fraction; RVEF, RV ejection fraction; ESVi, end-systolic volume index; EDVi, end-diastolic volume index; SV, stroke volume; ED, end-diastolic.

response, increase in heart rate ( $>10$ beats $/ \mathrm{min})$ and at least one adenosine-related symptom (chest tightness, shortness of breath, flushing, transient headache or nausea). There was a drop of systolic blood pressure $(>10 \mathrm{mmHg})$ in 10 patients (50\%). All side effects dissipated within a minute of stopping the adenosine infusion.

OS-CMR acquisition and optimization of the RV signal We found that the feasibility of detecting the RV OS-CMR signal differed according to the RV myocardial segments. 

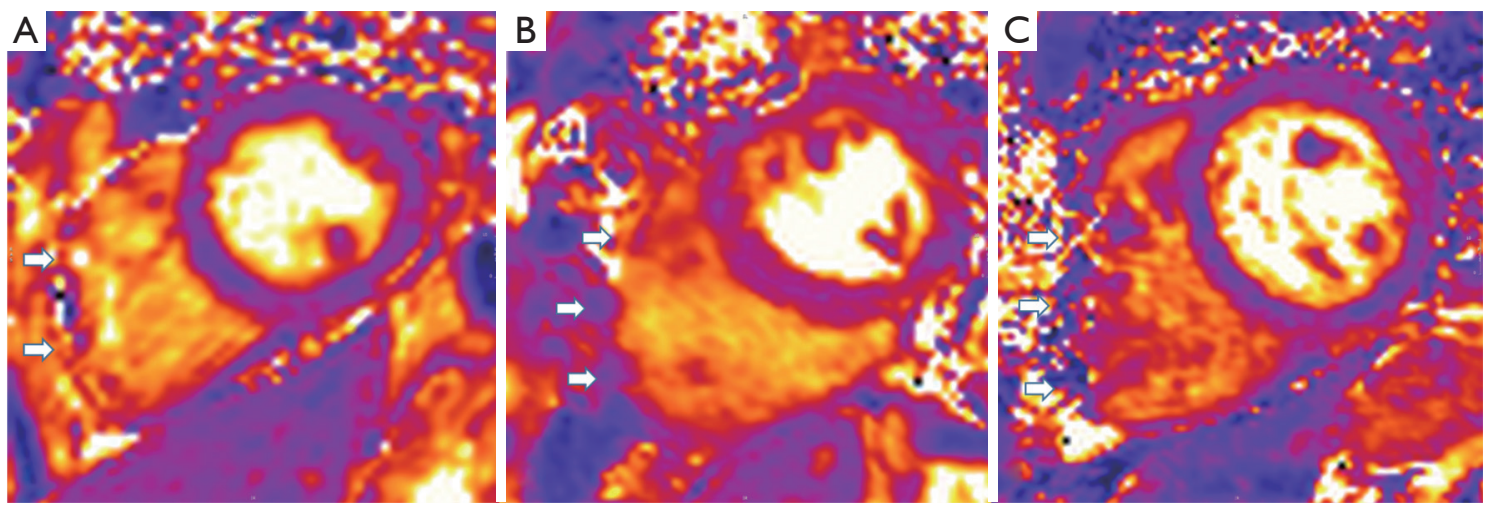

Figure 3 OS-CMR images in patients with pulmonary hypertension demonstrating poor image quality and segmental (RV Ant and RV FW) exclusions. Myocardial segments are excluded (arrows) from analysis primarily due to thin myocardium (A), off-resonance artefacts (B) and image artefacts (C). OS, oxygen-sensitive; CMR, cardiovascular magnetic resonance; RV Ant, right ventricle anterior; RV FW, right ventricle free-wall.

In both PAH and controls, reliable OS signal was only obtained in the inferior RV myocardial segment. Overall OS-CMR analysis was possible in $89 \%(396 / 444)$ of the $\mathrm{RV}$ (OS-CMR rest and stress) myocardial segments in the pulmonary hypertension patients and $80 \%$ (189/237) of the RV (OS-CMR rest and stress) myocardial segments in the control group. In the PAH group, $84 \%$ of the RV Ant (124/148) and RV FW (125/148) myocardial segments were analysed and $99 \%$ of RV $\operatorname{Inf}(147 / 148)$ myocardial segments were analysed. In the control group $73 \%$ of the RV Ant (58/79) and $75 \%$ of RV FW (59/79) myocardial segments were analysed with all the RV Inf myocardial segments were analysed. Segments were excluded from analysis because of thinned myocardium, off-resonance artefacts and image artefacts (Figure 3). We had attempted to reduce the limitation of thinned RV myocardium by reviewing all the SA cine acquisitions and selecting the best mid ventricle SA cine slice with the maximal RV myocardial thickness. The OS sequence was then applied to this mid ventricle SA slice location.

To limit the effects of off-resonance artefacts, a frequency shift scout series was applied to the selected mid ventricle SA cine slice location. A frequency range between -150 to $150 \mathrm{~Hz}$ was acquired and reviewed to select the optimal frequency. The delta frequency of the OS-CMR sequence was adjusted according to the optimal frequency chosen from the frequency shift scout. Image artefacts of the RV myocardium, in particular the RV FW segments were due to artefacts from air, heart-lung borders, sternal wires. This was overcome by modifying the adjustment volumes to match to selected mid ventricle SA slice location
(Figure 4, green box). To begin with the thickness of the adjusted volume was modified to focus only on the selected mid ventricle SA slice thickness (Figure $4 A$ ). Then the area of the adjustment volume was reduced inplane to the myocardium and to exclude unwanted air and sternal wires (Figure 4B). No adjustments were made to the matrix, field of view or slice thickness between rest and stress OS-CMR imaging in both groups. All the OS-CMR images were acquired in the same part of the cardiac cycle. A constant OS-CMR sequence voxel size of $2.02 \mathrm{~mm} \times 1.77 \mathrm{~mm}$ was maintained in both PAH and controls.

\section{Oxygenation response (OS-CMR signal)}

The RV OS SI response in the three RV segments in PAH patients were: RV Ant $10 \% \pm 12 \%$, RV FW $3 \% \pm 15 \%$ and RV Inf $11 \% \pm 9 \%$. In controls, reliable OS SI response was obtained in the RV Inf segment. The RV Inf OS SI changes between $\mathrm{PAH}$ patients and controls was $11 \% \pm 9 \%$ vs. $17 \% \pm 5 \%(\mathrm{P}=0.045)$. Figure $5 \mathrm{~A}$ further highlights the distribution of OS signal response in $\mathrm{PAH}$ vs. control group to stress in the inferior RV segments. There was less variability of the OS SI between PAH patients and controls. In the RV Inf, the PAH patients have a median OS SI change of $11 \%$ (interquartile range, $5 \%$ to $18 \%$ ) versus control median OS SI change of $17 \%$ (interquartile range, $12 \%$ to $21 \%)$. The inter-observer coefficient variability was 0.812 and the intra-observer coefficient variability was 0.945 .

\section{LV myocardial oxygenation response (OS-CMR)}

The global LV $\triangle$ OS-CMR SI change was significantly lower 

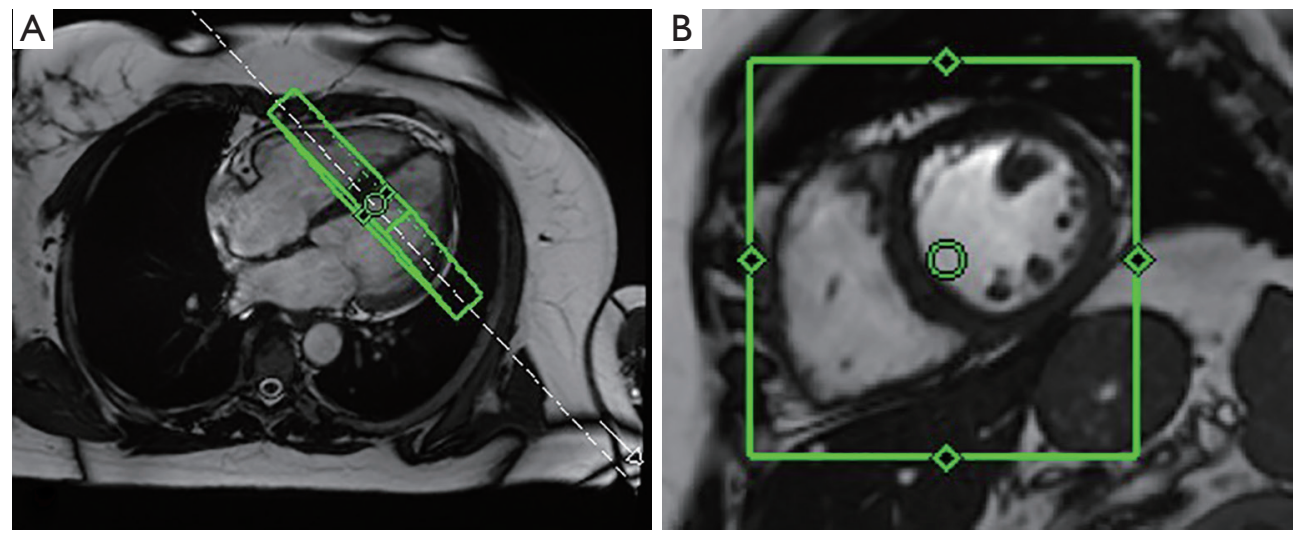

Figure 4 Adjustments volumes made to OS-CMR cardiac shim images to mitigate the effects of artefacts. Adjusted volume modified to focus on the selected mid ventricle SA slice thickness (A). Adjustment volume reduced inplane to the myocardium and to exclude unwanted air and sternal wires (B). Green box indicates selected mid ventricle SA slice location. OS, oxygen-sensitive; CMR, cardiovascular magnetic resonance; $\mathrm{SA}$, short axis.

in the PAH group compared to the controls $(11.1 \% \pm 7.3 \%$ vs. $21.1 \% \pm 9.1 \%, \mathrm{P}=0.019$ ) (Figure $5 B$ ).

\section{Relationship between $R V$ myocardial oxygenation, $L V$ myocardial oxygenation and CMR volumetric and functional indices}

There was a strong correlation between RV OS-CMR SI and LV OS-CMR SI $(\mathrm{r}=0.86, \mathrm{P}<0.001)$ (Figure $6 A)$. Furthermore there was an inverse relation whereby the RV Inf OS SI change was increasingly blunted with increasing $\mathrm{RV}$ wall thickness $(\mathrm{r}=-0.76, \mathrm{P}<0.001)$ (Figure $6 B)$. However there was no correlation between RV Inf OS SI and CMR RVEF ( $\mathrm{r}=0.24, \mathrm{P}=0.346)$, CMR RV EDVi $(\mathrm{P}=-0.21$, $\mathrm{P}=0.377)$ or $\mathrm{RV}$ mass index $(\mathrm{r}=-0.44, \mathrm{P}=0.079)$.

\section{Discussion}

The main finding of this proof of concept study is that the use of OS-CMR with vasodilator stress is safe and feasible in PAH patients. We found that compared with agematched healthy volunteers, OS-CMR shows myocardial deoxygenation most reliably in the inferior $\mathrm{RV}$ of patients with PAH. Furthermore, the RV myocardial deoxygenation correlates with the LV myocardial deoxygenation in $\mathrm{PAH}$ patients. This technique therefore holds promise as a noninvasive tool to assess early RV myocardial ischemia in patients with PAH.

\section{Patbological mechanisms of $R V$ ischemia in PAH}

It has long been recognised that an ischaemic insult is the most likely precipitant in the remodelling and subsequent development of RV dysfunction in PAH (4). This occurs in one of many forms. (I) Increasing pulmonary vascular and thence RV pressure decreases the pressure gradient between the aorta and the RV and might thereby reduce myocardial blood flow in the $\mathrm{RV}$ branch of the right coronary artery, which may lead to ischemia $(16,17)$. Progressive RV hypertrophy (RVH) without a sufficient compensatory increase in the myocardial vasculature and flow would likely exacerbate ischemia of the RV (18-20). It has been demonstrated in the advanced stages of PAH that right coronary perfusion pressure can drop below $50 \mathrm{mmHg}$ leading to a decline of RV contractile function (21). (II) Microvascular ischemia may also occur, due to occlusive microvascular damage and impairment in angiogenesis (known as capillary rarefaction); this has been demonstrated in animal models and SSc associated PAH $(17,21)$. There is also decreased expression of genes such as insulin growth factor 1, VEGF, apelin and angiopoietin-1 in the RV in PAH, which can lead to further microvascular damage, rendering the myocardial fibres more vulnerable to ischemia (2). (III) Dysfunctional mitochondrial metabolism has been another pathologically relevant feature of myocardial ischemia demonstrated in hypertrophied RV of PAH. In animal models of PAH with RVH, there is a shift from fatty acid oxidation to glycolysis as a preference 
A

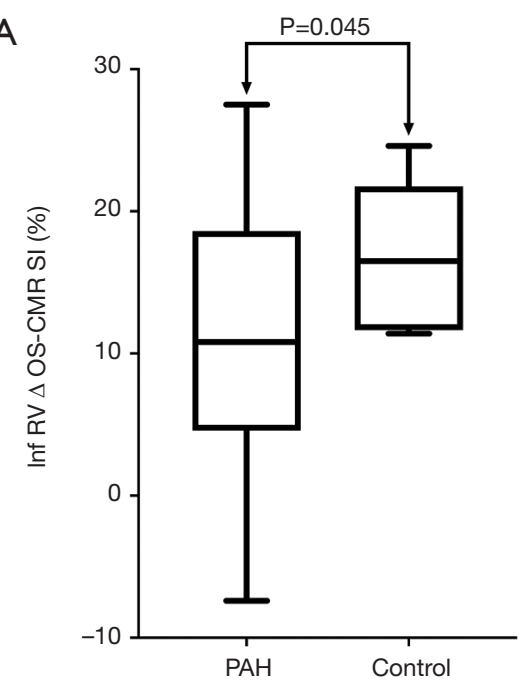

B

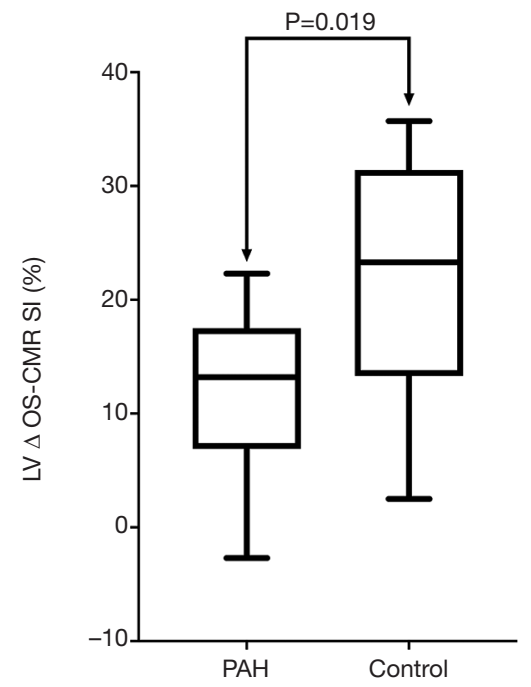

Figure 5 Distribution of OS signal response to stress between PAH and control in the inferior RV segment (A) and LV (B). OS, oxygensensitive; $\mathrm{PAH}$, pulmonary arterial hypertension; $\mathrm{RV}$, right ventricle; $\mathrm{LV}$, left ventricle.

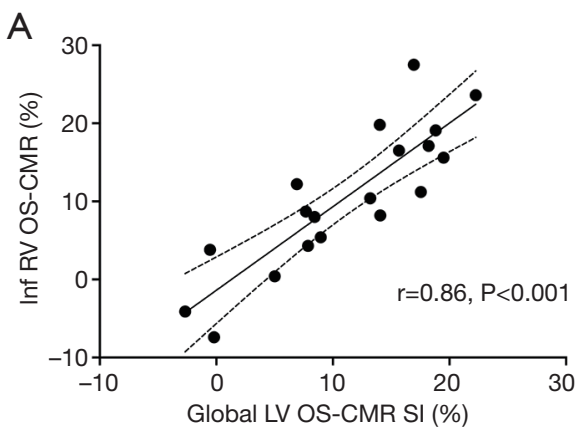

B

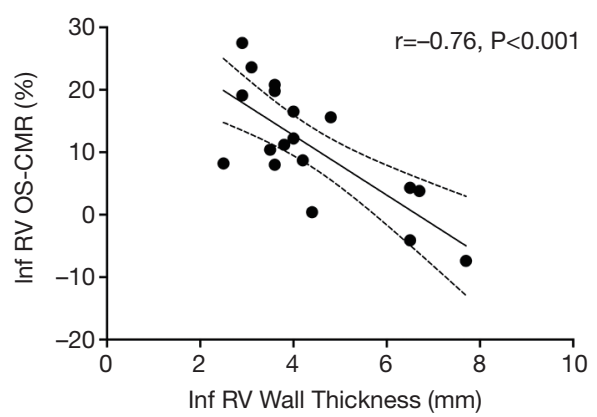

Figure 6 Correlation between Inf RV OS-CMR SI (\%) to global LV OS-CMR SI (\%) (A) and Inf RV wall thickness (mm) (B). OS, oxygensensitive; CMR, cardiovascular magnetic resonance; RV, right ventricle; LV, left ventricle; SI, signal intensity.

of energy substrates (22). This shift in glycolysis has been demonstrated by increased uptake of fluorodeoxyglucose on positron emission tomography (PET) in PAH patients with RVH (23). This metabolic shift to aerobic glycolysis has been associated with decreased cardiac output and RV contractility $(22,24)$. The complex interplay between reduced right coronary perfusion pressure, microvascular ischemia and worsening RV contractility can lead to a decrease in myocardial oxygenation. Even in the absence of epicardial coronary artery disease, these mechanisms could form a vicious cycle leading to progressive RV dysfunction, resulting in RV hibernation (25).

\section{Feasibility of $O S-C M R$ in the $R V$}

The use of OS-CMR has been established as a direct assessment of myocardial oxygenation in the LV (6). The OS SI changes reflect the changes in haemoglobin oxygenation; hence it can be used to study the state of myocardial oxygenation in the capillary bed. OS-CMR is a novel non-invasive technique that can provide insights into the balance (or the lack of) between myocardial oxygen supply and demand. As it is a direct measure of oxygenation changes within the myocardium, it has been utilized to demonstrate ischemia in diseases in the absence of significant epicardial coronary artery disease (6). The OS-CMR technique has increased understanding of in vivo myocardial oxygenation in epicardial coronary artery disease and cardiomyopathies involving the LV; however, its utility in the assessment of myocardial ischemia of RV has not yet been examined. 
Our study has demonstrated that OS-CMR of the $\mathrm{RV}$ with stress induced via pharmacological adenosine infusion is a safe and feasible technique in patients with PAH. Nevertheless, in some patients the RV Ant and RV FW segments had to be excluded from analysis due to thin myocardium (causing partial volume effects), off-resonance artefacts and image artefacts. Whilst every effort was taken to limit these effects, there are a known limitation of the OS-CMR technique. Other OS-CMR studies involving the $\mathrm{LV}$ have excluded segments with thinned myocardium and artefacts (from partial volumes) as it affects the accuracy of OS-CMR SI (26). In our study the measured Inf RV wall thickness and consistent voxel-size during OS-CMR imaging provided sufficient coverage to avert partial volume effects. Off-resonance artefacts seen in OS-CMR by nature arise from the inhomogeneities of using a higher magnetic field (27). However the advantages of using a higher field strength are improved ability to detect OS-CMR SI changes, increased contrast to noise ratio and signal to noise ratio (27). Despite these limitations, the feasibility of OSCMR in the RV offers a potentially novel non-invasive, contrast and tracer independent technique that may offer new insights to myocardial oxygenation in the RV. Furthermore the OS SI in the RV has a good correlation with the LV, an area of the myocardium in which the OSCMR technique has been extensively validated and studied $(5,6)$. Coincidentally we have demonstrated LV myocardial deoxygenation in PAH patients. While the focus of this study has been the feasibility of OS-CMR of the RV, the presence of $L V$ deoxygenation in $\mathrm{PAH}$ needs further study. In summary, establishing a means of studying the in-vivo state of myocardial oxygenation of the RV in PAH patients may help in the pathophysiological understanding of RV dysfunction in this high risk group.

\section{Study limitations}

This study has a small sample size which may increase the margin of error. Further, larger studies would help further optimize as well as determine the clinical utility of OSCMR of the RV in PAH and its association with clinical outcomes. Additionally, there was incomplete coverage of the full RV myocardium due to the CMR acquisition being performed on a single mid-ventricular slice however this is unlikely to be of major relevance in RV assessment of $\mathrm{PAH}$ which is a global process. Indeed, the mid RV wall is the most vulnerable to ischemia anatomically because of its precarious blood supply. The presence of thin myocardium, off-resonance artefacts and image artefacts led to poor image quality and segment exclusion. However, the feasibility and safety of pharmacological induced OSCMR of the RV should provide novel insights into the mechanisms of RV dysfunction in PAH patients. Moreover, a novel marker of early or impending RV failure might become a clinically useful guide for therapy augmentation. These findings should be considered as hypothesisgenerating for future studies.

\section{Conclusions}

This is the first study to show that pharmacological induced OS-CMR is a feasible and safe technique to identify and study myocardial oxygenation in the RV of PAH patients. With a larger study this technique has potential implications in the understanding of myocardial ischemia of the RV in patients with $\mathrm{PAH}$, or other pathologic conditions affecting the RV myocardium.

\section{Acknowledgments}

This research is being funded by the National Health and Medical Research Council of Australia and Actelion Pharmaceuticals Australia.

\section{Footnote}

Conflicts of Interest: The authors have no conflicts of interest to declare.

Ethical Statement: The authors are accountable for all aspects of the work in ensuring that questions related to the accuracy or integrity of any part of the work are appropriately investigated and resolved. This study was approved by the Southern Adelaide Clinical Human Research Ethics Committee (HREC/15/SAC/397), and all participants provided written and informed consent to participate in the study.

\section{References}

1. McLaughlin VV, McGoon MD. Pulmonary arterial hypertension. Circulation 2006;114:1417-31.

2. Gomez A, Bialostozky D, Zajarias A, et al. Right ventricular ischemia in patients with primary pulmonary hypertension. J Am Coll Cardiol 2001;38:1137-42.

3. D'Alonzo GE, Barst RJ, Ayres SM, et al. Survival in 
patients with primary pulmonary hypertension. Results from a national prospective registry. Ann Intern Med 1991;115:343-9.

4. Ryan JJ, Archer SL. Emerging concepts in the molecular basis of pulmonary arterial hypertension: part I: metabolic plasticity and mitochondrial dynamics in the pulmonary circulation and right ventricle in pulmonary arterial hypertension. Circulation 2015;131:1691-702.

5. Thulborn KR, Waterton JC, Matthews PM, et al. Oxygenation dependence of the transverse relaxation time of water protons in whole blood at high field. Biochim Biophys Acta 1982;714:265-70.

6. Sree Raman K, Nucifora G, Selvanayagam JB. Novel cardiovascular magnetic resonance oxygenation approaches in understanding pathophysiology of cardiac diseases. Clin Exp Pharmacol Physiol 2018;45:475-80.

7. Pauling L, Coryell CD. The magnetic properties and structure of hemoglobin, oxyhemoglobin and carbonmonoxyhemoglobin. Proc Natl Acad Sci U S A 1936;22:210-6.

8. Grover S, Lloyd R, Perry R, et al. Assessment of myocardial oxygenation, strain, and diastology in MYBPC3-related hypertrophic cardiomyopathy: a cardiovascular magnetic resonance and echocardiography study. Eur Heart J Cardiovasc Imaging 2019;20:932-8.

9. Shah R, Parnham S, Liang Z, et al. Prognostic Utility of Oxygen-Sensitive Cardiac Magnetic Resonance Imaging in Diabetic and Nondiabetic Chronic Kidney Disease Patients With No Known Coronary Artery Disease. JACC Cardiovasc Imaging 2019;12:1107-9.

10. Karamitsos TD, Arnold JR, Pegg TJ, et al. Patients With Syndrome X Have Normal Transmural Myocardial Perfusion and Oxygenation. Circ Cardiovasc Imaging 2012;5:194-200.

11. Parnham S, Gleadle JM, Bangalore S, et al. Impaired myocardial oxygenation response to stress in patients with chronic kidney disease. J Am Heart Assoc 2015;4:e002249.

12. Karamitsos TD, Leccisotti L, Arnold JR, et al. Relationship Between Regional Myocardial Oxygenation and Perfusion in Patients With Coronary Artery Disease. Circ Cardiovasc Imaging 2010;3:32-40.

13. Arnold JR, Karamitsos TD, Bhamra-Ariza P, et al. Myocardial oxygenation in coronary artery disease: insights from blood oxygen level-dependent magnetic resonance imaging at 3 Tesla. J Am Coll Cardiol 2012;59:1954-64.

14. Cerqueira MD, Weissman NJ, Dilsizian V, et al. Standardized myocardial segmentation and nomenclature for tomographic imaging of the heart. A statement for healthcare professionals from the Cardiac Imaging Committee of the Council on Clinical Cardiology of the American Heart Association. Circulation 2002;105:539-42.

15. Sharma P, Socolow J, Patel S, et al. Effect of Gd-DTPABMA on blood and myocardial $\mathrm{T} 1$ at $1.5 \mathrm{~T}$ and $3 \mathrm{~T}$ in humans. J Magn Reson Imaging 2006;23:323-30.

16. Bogaard HJ, Abe K, Vonk Noordegraaf A, et al. The right ventricle under pressure: cellular and molecular mechanisms of right-heart failure in pulmonary hypertension. Chest 2009;135:794-804.

17. Piao L, Fang YH, Parikh K, et al. Cardiac glutaminolysis: a maladaptive cancer metabolism pathway in the right ventricle in pulmonary hypertension. J Mol Med (Berl) 2013;91:1185-97.

18. Akasaka T, Yoshikawa J, Yoshida K, et al. Comparison of relation of systolic flow of the right coronary artery to pulmonary artery pressure in patients with and without pulmonary hypertension. Am J Cardiol 1996;78:240-4.

19. Murray PA, Vatner SF. Reduction of maximal coronary vasodilator capacity in conscious dogs with severe right ventricular hypertrophy. Circ Res 1981;48:25-33.

20. van Wolferen SA, Marcus JT, Westerhof N, et al. Right coronary artery flow impairment in patients with pulmonary hypertension. Eur Heart J 2008;29:120-7.

21. Bogaard HJ, Natarajan R, Henderson SC, et al. Chronic pulmonary artery pressure elevation is insufficient to explain right heart failure. Circulation 2009;120:1951-60.

22. Piao L, Sidhu VK, Fang YH, et al. FOXO1-mediated upregulation of pyruvate dehydrogenase kinase-4 (PDK4) decreases glucose oxidation and impairs right ventricular function in pulmonary hypertension: therapeutic benefits of dichloroacetate. J Mol Med (Berl) 2013;91:333-46.

23. Oikawa M, Kagaya Y, Otani H, et al. Increased [18F] fluorodeoxyglucose accumulation in right ventricular free wall in patients with pulmonary hypertension and the effect of epoprostenol. J Am Coll Cardiol 2005;45:1849-55.

24. Piao L, Fang YH, Cadete VJ, et al. The inhibition of pyruvate dehydrogenase kinase improves impaired cardiac function and electrical remodeling in two models of right ventricular hypertrophy: resuscitating the hibernating right ventricle. J Mol Med (Berl) 2010;88:47-60.

25. Piao L, Marsboom G, Archer SL. Mitochondrial metabolic adaptation in right ventricular hypertrophy and failure. J Mol Med (Berl) 2010;88:1011-20.

26. Fischer K, Yamaji K, Luescher S, et al. Feasibility of cardiovascular magnetic resonance to detect oxygenation deficits in patients with multi-vessel coronary artery 
disease triggered by breathing maneuvers. J Cardiovasc Magn Reson 2018;20:31.

27. Dharmakumar R, Arumana JM, Tang R, et al. Assessment of regional myocardial oxygenation changes in the

Cite this article as: Sree Raman K, Stokes M, Walls A, Perry R, Steele PM, Burdeniuk C, De Pasquale CG, Celermajer DS, Selvanayagam JB. Feasibility of oxygen sensitive cardiac magnetic resonance of the right ventricle in pulmonary artery hypertension. Cardiovasc Diagn Ther 2019;9(5):502-512. doi: 10.21037/cdt.2019.09.19 presence of coronary artery stenosis with balanced SSFP imaging at 3.0 T: theory and experimental evaluation in canines. J Magn Reson Imaging 2008;27:1037-45. 